\title{
Quantum Mechanical Simulations of Electronic Stopping in Metals
}

\author{
D.R. Mason ${ }^{\mathrm{a}, *}$, C.P. Race ${ }^{\mathrm{b}}$, W.M.C. Foulkes ${ }^{\mathrm{a}, *}$, M.W. Finnis ${ }^{\mathrm{a}, \mathrm{c}}$, A.P. \\ Horsfield $^{\mathrm{a}, \mathrm{c}}$, A.P. Sutton ${ }^{\mathrm{a}}$ \\ ${ }^{a}$ Department of Physics, Imperial College London, SW7 2AZ, UK \\ ${ }^{b}$ Max-Planck Institute for Iron Research, 40237 Düsseldorf, Germany \\ ${ }^{c}$ Department of Materials, Imperial College London, SW7 2AZ, UK
}

\begin{abstract}
The close spacing of electron energy levels at the Fermi surface of a metal allows for a ready exchange of energy between ionic and electronic subsystems. In molecular dynamics (MD) simulations of fast moving ions, the heat transfer to electrons is sometimes modelled as a frictional force that slows the ions. Quantum mechanical simulations lay bare these processes and reveal how best to characterise electronic friction and heating for direct incorporation into MD. In this paper, we discuss the limitations of the description of electronic damping as a viscous force, the validity of the two-temperature model, and how the nonadiabatic movement of electrons between bonds leads to directional stopping.
\end{abstract}

Keywords: Electronic stopping, computer simulation, metals

Metals differ from insulators in that they have a finite density of electronic energy states at the Fermi level. The existence of large numbers of possible electronic excitations at low energy allows for a constant interchange of small amounts of energy between ionic and electronic degrees of freedom. In equilibrium, when there is no net flow of energy between ions and electrons, the fluctuations in the ionic energy resulting from this interchange are small. Furthermore, since the thermal energy scale $k T$ is normally much smaller than the Fermi energy $E_{F}$, the electronic subsystem remains close to its ground state and the metal may be successfully modelled using the Born-Oppenheimer separation. When the ionic and electronic sub-systems are far from equilibrium, however, the exchange of energy between them can be important. In radiation damage, sputtering, or ion channelling, the high-energy ions lose a significant fraction of their energy to the colder electron gas $[1,2,3,4,5,6]$, a process

\footnotetext{
${ }^{*}$ Corresponding author

Email addresses: daniel.mason@imperial.ac.uk (D.R. Mason), wmc.foulkes@imperial.ac.uk (W.M.C. Foulkes)
} 
known as electronic stopping. In a current-carrying wire, by contrast, the excited electrons lose energy to the ions by generating phonons $[7,8]$.

Recent molecular dynamics (MD) simulations of radiation damage cascades $[9,10]$ have shown that electronic stopping can promote or inhibit the rate of defect production, depending on the strength of the coupling between ions and electrons. As the ion-electron coupling strength is increased from zero, the rate at which the ions lose energy to electrons rises, enhancing the rate of cooling of the cascade and so quenching in defects. At high enough coupling strength, the electronic system is heated more rapidly than the electrons can carry the heat away. The electrons then act as a thermal reservoir, allowing defects to anneal. Sputtering simulations $[4,5]$ have shown that electronic heating can lead to a hot spot forming at the surface centred on the impact, strongly affecting secondary ion formation.

In the standard picture of electronic stopping, an ion moves in a homogeneous electron gas $[11,12]$. The electrons take a finite time to respond to the movement of the ion and the electronic screening cloud lags the ionic motion, producing Coulomb forces that pull the ion back and slow it down. The energy transfer from ions to electrons is described to first order by the imaginary part of the inverse of the frequency-dependent dielectric function, which is known as the electron loss function. In 1954, Lindhard showed how, in the low ionic velocity limit of this model, the electrons provide a viscous drag on a moving ion, proportional to and anti-parallel to the velocity.

But this physical picture of an electronic ether is at odds with the standard MD picture, where forces depend on the instantaneous positions of all the atoms. Surely, in reality, the lagged electron currents induced by the ionic motion strengthen some bonds and weaken others, producing directional forces that may not always oppose the velocity. This paper discusses results obtained using a more chemically realistic approach to electronic stopping and explains how they suggest better methods for including electronic effects in MD simulations.

\section{A quantum mechanical description of ion dynamics}

A complete theoretical treatment of the coupling between ions and electrons requires a quantum mechanical description of both electrons and phonons. Computations based on such theories are currently too expensive to allow direct simulation of the dynamics of more than a handful of atoms. Fortunately, the energy transfer from hot ions to cold electrons can be accurately captured using an approximate scheme known as Ehrenfest dynamics [13]. In this approach, the ions are treated as classical point-like particles, as in MD, but experience forces that depend on the instantaneous electronic state, obtained by solving the time-dependent Schrödinger equation [14]. (This is in contrast to Car-Parrinello dynamics [15], where the Born-Oppenheimer separation of time scales is used to justify the assumption that the electrons remain in their ground state at all times.) The difference between the evolved Ehrenfest electronic state and the electronic ground state accumulates over time. The energy of the electrons therefore rises steadily while that of the ions reduces. 
Quantitative simulations of electronic stopping based on time-dependent density functional theory (TDDFT) are feasible in some restricted circumstances $[16,17]$, but it is not yet possible to model the dynamics of a realistic cascade. If the cascade energy is high, the damaged region grows rapidly and a large simulation is required. If the cascade energy is low, the individual energy transfers from ions to electrons are very small - of order meV for a keV ion - and the spectrum of available electronic excitations must be very dense. This again requires the simulation to be large. In fact, a system containing thousands of atoms is required to model even a $1 \mathrm{keV}$ cascade [18]. To make simulations on this scale possible, we have used a very simple s-band tight-binding Hamiltonian, with parameters fitted to reproduce the mechanical properties of copper [19]. The simplicity of the model aids interpretation of the results and makes it easier to identify the relevant physical processes, but the electronic bandstructure of the model Hamiltonian differs substantially from that of real copper. This means that the results obtained, although they provide a great deal of qualitative understanding, are not quantitatively accurate.

Consider a system of $N$ classical atoms, all assumed to be of mass $M$ for simplicity. The atomic positions are described by the $3 N$-dimensional vector $\vec{R}$ and their momenta by the $3 N$-dimensional vector $\vec{P}$. The electronic wavefunctions and occupations can be compactly represented in terms of the single-particle density operator,

$$
\hat{\rho}(t)=\sum_{i} f_{i}\left|\phi_{i}(t)\right\rangle\left\langle\phi_{i}(t)\right|,
$$

where $f_{i}$ is the occupation of orbital $\phi_{i}(t)$. The orbital occupations are set at time $t=0$ and depend on the initial temperature, but remain constant throughout the Ehrenfest simulation. The wavefunctions $\phi_{i}(t)$ evolve in the timedependent potential of the moving atoms according to the time-dependent Schrödinger equation. The energy of the coupled system is written as

$$
E=\frac{|\vec{P}|^{2}}{2 M}+U(\vec{R})+\operatorname{Tr}(\hat{\rho} \hat{H}(\vec{R})),
$$

where $U(\vec{R})$ is a repulsive energy acting between ions, $\hat{H}$ is the tight-binding Hamiltonian, ${ }^{1}$ and the trace gives the attractive "band" energy arising from electronic bonding [20]. The form of this expression is reminiscent of that used in second-moment empirical models of inter-atomic forces such as the FinnisSinclair potential [21], which can be regarded as providing a specific approximation to the final term. The equations of motion of the Ehrenfest method,

$$
\frac{\mathrm{d} \vec{R}}{\mathrm{~d} t}=\frac{\vec{P}}{M},
$$

\footnotetext{
${ }^{1}$ A self-consistent mean-field treatment of electron-electron interactions within the Hartree approximation can be added to the equations without significantly affecting computational speed [14, 18]. Hartree terms were included in most of our simulations, but screening is very efficient in metals and they have little effect on the non-adiabatic transfer of energy from ions to electrons. For the sake of clarity, we have omitted them from this discussion.
} 


$$
\begin{aligned}
\frac{\mathrm{d} \vec{P}}{\mathrm{~d} t} & =-\nabla_{\vec{R}} U(\vec{R})-\operatorname{Tr}\left(\hat{\rho} \nabla_{\vec{R}} \hat{H}\right), \\
\frac{\mathrm{d} \hat{\rho}}{\mathrm{d} t} & =\frac{\partial \hat{\rho}}{\partial t}=\frac{1}{i \hbar}[\hat{H}, \hat{\rho}],
\end{aligned}
$$

conserve the Ehrenfest energy $E$ as defined in Eq. (2) [14]. The last of these three equations, the quantum Liouville equation, describes how the density operator evolves if the wavefunctions from which it is constructed obey the timedependent Schrödinger equation. We thus have a set of quantum mechanical electrons evolving according to a Hamiltonian that depends on the positions of a set of classical ions. The ions satisfy the laws of Newtonian dynamics, under the influence of forces explicitly dependent on the time-evolved electronic state.

\section{Electronic Friction in MD}

The simplest way to incorporate electronic stopping into an MD simulation is to treat the electrons as a viscous fluid imparting a frictional force $-\beta \vec{V}_{a}$, where $V_{a}$ is the velocity of atom $a$ and $\beta$ is the drag coefficient. This approach can be justified using the Fermi-Teller picture, Firsov's model of binary collisions [22], or Lindhard and Scharff's scattering formula [23]. Finnis et al. [24] derive a form for $\beta$ based on the expected rate of energy transfer between ionic and electronic subsystems. Nordlund et al. [25] use a coefficient $\beta$ taken from the SRIM code [26], but assume that electronic drag only affects atoms with kinetic energy above a threshhold, taken to be $10 \mathrm{eV}$. Caro and Victoria [27] extend the drag model by noting that $\beta$ for a given atom must be a function of the local electron density.

We have used the Ehrenfest dynamics method described above to compare these approximations. Over a large number of representative low-energy cas-

cades (see figure 1), the energy passed from ions to electrons for a given classical model is computed as an integral along the ionic trajectories:

$$
\Delta E_{\text {model }}(t)=\int_{0}^{t} \sum_{a} \beta_{a}(\vec{R}(\tau))\left|\vec{V}_{a}(\tau)\right|^{2} \mathrm{~d} \tau
$$

We can compare this to the Ehrenfest energy transfer

$$
\Delta E_{\mathrm{Ehr}}(t)=\operatorname{Tr}(\hat{H}(\vec{R}(t)) \hat{\rho}(t))-\operatorname{Tr}\left(\hat{H}(\vec{R}(t)) \hat{\rho}_{0}(\vec{R}(t))\right),
$$

where $\hat{\rho}_{0}(\vec{R})$ is a suitably chosen electronic density operator for a system in which the ions are fixed at $\vec{R}$. If the initial electronic temperature is assumed to be zero, $\hat{\rho}_{0}(\vec{R})$ is the ground-state density operator for ionic configuration $\vec{R}$; if the initial electronic temperature is finite, $\hat{\rho}_{0}(\vec{R})$ is the equilibrium density operator in the canonical ensemble with the ions frozen at $\vec{R}$.

The two measures of energy transfer can be compared using standard linear regression to evaluate the quality of fit of the empirical model [28]. For a low electronic temperature, we found that a single viscous damping coefficient does 


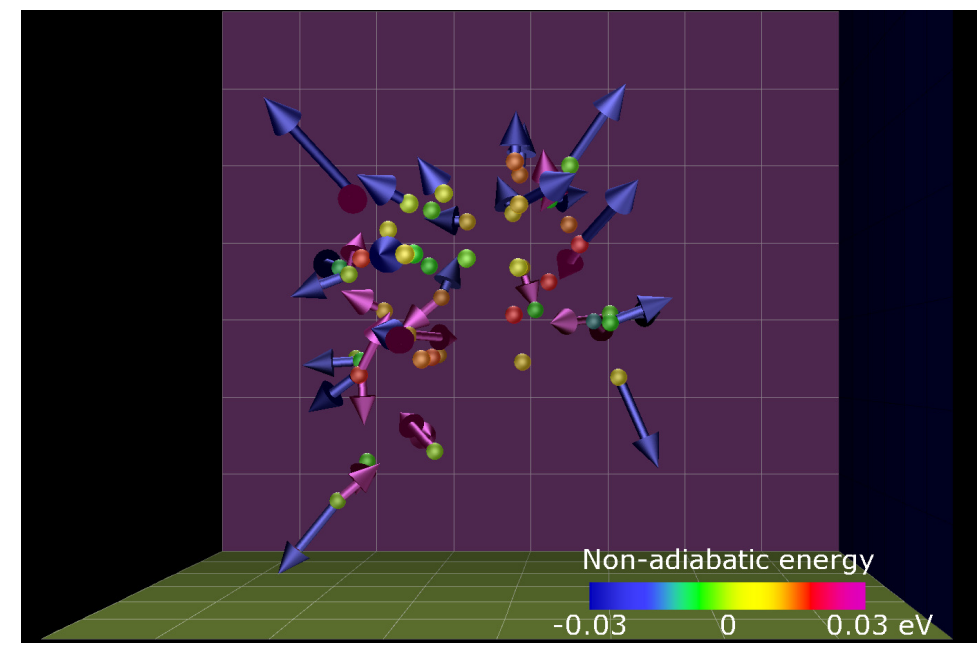

Figure 1: A snapshot $30 \mathrm{fs}$ into a $1 \mathrm{keV}$ cascade simulated with Ehrenfest dynamics. Ions moved $>0.1$ Afrom their original positions are coloured according to their non-adiabatic energy $\Delta E_{a}=\left\langle a\left|\hat{H}\left(\hat{\rho}-\hat{\rho}_{0}\right)\right| a\right\rangle$, where $|a\rangle$ is an orbital centred on atom $a$ ( see text ). Velocity is indicated by a blue arrow, and the non-adiabatic force $\Delta f_{a}=\operatorname{Tr}\left(\nabla_{a} \hat{H}\left(\hat{\rho}-\hat{\rho}_{0}\right)\right)$ by a pink arrow. Statistics from dozens of simulations like this reveal the extent to which forces due to electronic excitation can be approximated by a viscous damping.

a surprisingly good job of capturing the total energy transfer, as shown in figure 2(a). The addition of an environmental dependence, as suggested by Caro and Victoria [27], further improves this result (see figure 2(b)). We found no support for a low energy cutoff on ions which are damped. This is an important result because it implies that the total rate of energy transfer from ions to electrons does not decrease as the cascade proceeds and the initial energy is spread over more and more ions. The ions continue to lose energy to the electrons until the ionic and electronic systems reach similar temperatures. Consequently, a large amount of the initial kinetic energy can be lost from phonon-like modes, heating the electrons in the periphery and late stages of a cascade [29].

\section{Electronic Temperature}

Ehrenfest simulations provide no mechanism for the electrons to thermally equilibrate with each other, and so the electronic energy distribution at the end of a simulation need not be thermal. In practice, however, the motion of the atoms produces a large number of very small electronic transitions, and the evolution of the occupation function of the electronic energy levels resembles a one-dimensional diffusion process in energy space. If the initial electronic temperature is zero, so that all states below the Fermi level are occupied and all states above the Fermi level are empty, this diffusive evolution leads to a distribution of occupations in the form of an error function. As shown in figure 3(a), the resulting electronic energy distribution closely resembles a thermal distribu- 


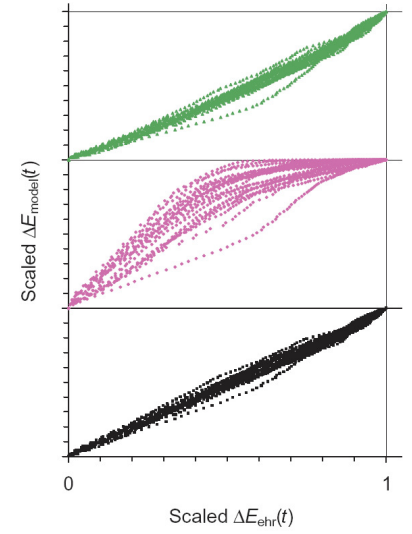

(a)

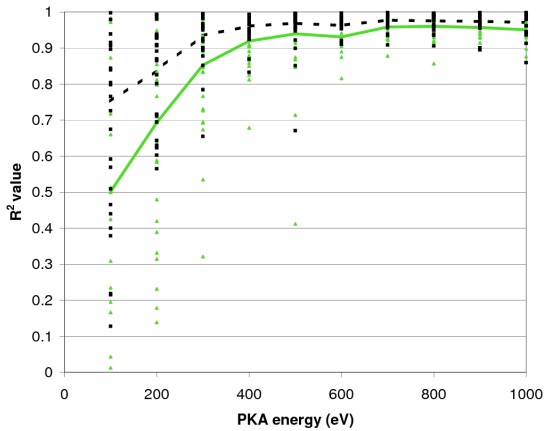

(b)

Figure 2: (a) Scatter plot comparing the non-adiabatic energy transfers calculated using the three classical models of electronic damping with the energy transfer calculated using the quantum mechanical Ehrenfest method. Points are plotted every 2.5fs for 24 cascades, each with a cascade energy of $100 \mathrm{eV}$. The upper plot is for a homogeneous damping coefficient; the middle plot uses a $10 \mathrm{eV}$ kinetic energy cut-off; and the bottom plot uses the electrondensity-dependent damping coefficient suggested by Caro and Victoria. Each axis is scaled by the energy transfer at $\mathrm{t}=200 \mathrm{fs}$. A straight line of gradient 1 would indicate a perfect correspondence between classical and Ehrenfest methods. (b) $\mathrm{R}^{2}$ measures of the goodness of fit for the homogeneous and density-dependent damping models for a range of cascade energies. Square symbols denote results for the Caro-Victoria model (dashed line to guide the eye), and triangles (solid line) are results for homogeneous damping. (Figure from ref [28].) 
tion, and a pseudo-temperature $T$ can readily be computed [30]. An accurately thermal-looking electron distribution has also been seen to be generated by sputtering [16] and femtosecond laser pulses [31].

Further backing for the idea of an electronic pseudo-temperature is shown in figure 3(b), which illustrates how the measured energy transfer may be reproduced by substituting the pseudo-temperature into the Sommerfeld heat capacity equation [30]. The pseudo-temperature $T$ is therefore close to the temperature of a reservoir that would exchange no energy on average with the excited electron gas. This lends support to the idea of using a two-temperature model in MD simulations where ionic and electronic subsystems are out of equilibrium.

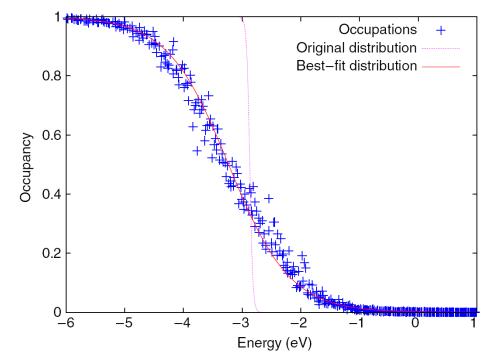

(a)

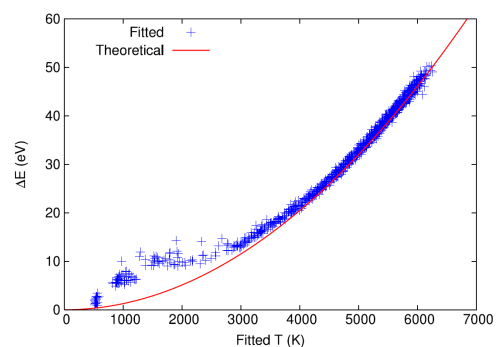

(b)

Figure 3: (a) The population of the instantaneous eigenstates 225fs into a typical cascade simulation. A Fermi-Dirac distribution corresponding to the original electronic temperature of $300 \mathrm{~K}$ is shown, along with a Fermi-Dirac function corresponding to a temperature of 6055 $\mathrm{K}$ as a best fit to the excited occupation distribution. (b) Plot of $\Delta E_{\mathrm{Ehr}}$ (equation (5)) against fitted temperature drawn from forty-four cascade simulations with the primary knock-on atom given an energy of $2 \mathrm{keV}$. The solid line indicates the theoretical curve for the electronic heat capacity based on the Sommerfeld expansion. At the earliest simulation times, only one or two atoms have moved and the electronic heating is too local to be modelled using a single temperature for the whole cell. The fitted temperature is thus too low at early times. (Figures from ref [30].)

The practical value of the concept of a pseudo-temperature suggests separating the electronic density operator obtained from the Ehrenfest time evolution into a ground-state contribution, a correction that describes the rise in temperature, and a remainder [30]: $\hat{\rho}(t)=\hat{\rho}^{0}+\left(\hat{\rho}^{T}-\hat{\rho}^{0}\right)+\left(\hat{\rho}(t)-\hat{\rho}^{T}\right)$. The $\hat{\rho}^{0}$ and $\hat{\rho}^{T}-\hat{\rho}^{0}$ terms depend on the ionic positions $\vec{R}$ and temperature $T$ but not on how those positions and temperature were reached. The corresponding contributions to the total energy are therefore conservative: if the ions are moved around a closed path at constant $T$, returning to their original positions, these contributions return to their original values. The total force on an ion can therefore be viewed as the sum of a conservative force appropriate for the zero temperature electronic ground state, a conservative correction due to the increase in electronic temperature, and a non-conservative correction due to the $\hat{\rho}(t)-\hat{\rho}^{T}$ term. 


\section{Directional Forces}

As discussed above, electronic friction is generally incorporated into MD simulations by the addition of a viscous damping force $-\beta \vec{V}$. Physical intuition and our Ehrenfest simulations suggest that this is sensible to some extent, but $\beta$ is certainly not a scalar constant. Firstly, as noted by Caro and Victoria [27], the strength of the damping is a function of the local electronic density. Secondly, at very high electronic temperatures, there is no preference for phonon absorption rather than emission; the ions are buffeted by electrons and undergo Brownian motion, but are not smoothly damped. Thirdly, if all of the atoms move with uniform velocity, so do the electrons and there is no damping; the value of $\beta$ therefore depends on relative, rather than absolute, velocities. Fourthly, where an impact between atoms is sufficiently energetic that it is possible to promote electrons from low-lying energy levels, band-structure effects are seen. Fifthly, the electronic excitation generated by moving ions accumulates over time, so the damping is history dependent. And lastly, the electronic excitations may strengthen some bonds whilst weakening others, so the non-adiabatic electronic force depends on the direction and nature of the bonding. To summarise: $\beta$ is really a tensor dependent on the positions and relative velocities of all ions and their history [32, 33, 18].

However, in large systems at least, it seems that the historical accumulation of electronic excitations contributes a non-adiabatic force with almost zero expectation value - the damping force is thus effectively time local. Moreover, the instantaneous rate of change of the difference between the Ehrenfest electronic density and the ground-state density depends primarily on the gradient of the Hamiltonian, which is spatially highly localised. We have recently derived an expression for the non-adiabatic force that can be expressed in terms of the second moments of the local density of states. Since these quantities are already computed in any simulation using a second-moment embedded atom empirical potential, our expression is suitable for direct incorporation within MD simulations [34]. Expressed in terms of the attractive potential energy of atom $a$, $\sqrt{\Phi_{a}}=\sqrt{\sum_{b} H_{a b}^{2}}$, as used in second-moment embedded atom models, this new force may be written

$$
\vec{F}_{a}=4 \hbar x \sum_{b} \sqrt{\frac{\bar{\Phi}}{\Phi_{a}^{2} \Phi_{b}}}\left(\nabla_{\vec{R}_{a}} H_{a b} \cdot\left(\vec{V}_{b}-\vec{V}_{a}\right)\right) \nabla_{\vec{R}_{a}} H_{a b}
$$

where $\bar{\Phi}$ is the average of the square of the attractive energy and $x$ an adjustable parameter to fit to experimental data. Electronic damping forces in the form of a viscous drag opposing the velocity appear when a single ion is moving down a channel in an otherwise stationary lattice. Otherwise, these non-adiabatic forces can be in any direction, depending on which bonds the excitation preferentially modifies. As shown in figure 4, this model faithfully reproduces in an MD simulation both the magnitude and direction of the non-adiabatic forces during ion collisions in a metal in a collision cascade. This is a much more stringent test than just capturing the mean energy transfer rate. 


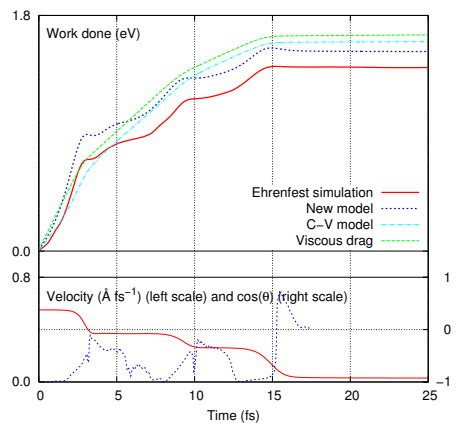

(a)

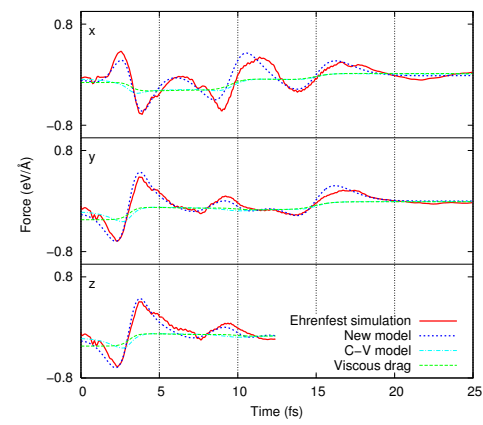

(b)

Figure 4: (a) The upper panel shows the work done by the non-adiabatic force on the primary knock-on atom in a sample Ehrenfest simulation of a collision cascade. In this case, the primary knock-on atom undergoes an oblique collision with the first atom that it encounters along its path. The lower panel shows the ionic velocity (solid line) and the cosine of the angle between the velocity and the non-adiabatic force in the Ehrenfest simulation (shown only where the velocity is appreciable). (b) The Cartesian components of the non-adiabatic force. In each case data are shown for the force derived from the Ehrenfest simulation, the force calculated using our new model, a simple viscous drag, and the density dependent drag of Caro and Victoria [27] (labelled C-V model). (Figure from ref [34].)

\section{Lattice Heating and Thermal Diffusion}

When the electrons have been heated, the next challenge is to describe the movement of this energy. Some heat will diffuse away, as the electronic thermal conductivity of metals is very high. Some heat will be returned to the ions through stimulated and spontaneous phonon emission. While both these processes can in principle be described using quantum mechanical models, they present significant challenges.

Perhaps surprisingly, it remains an open problem to establish Fourier's law of heat diffusion rigorously from quantum mechanics, although it has been possible to show the emergence of thermal diffusion in weakly coupled systems [35] and numerically [36]. MD simulations typically take a pragmatic approach, assuming that the electronic heat flow is described by Fourier's law and applying it to the electronic temperature field in a two-temperature model with an appropriate diffusion coefficient $[10,4]$. In this way, electronic heat can cross the boundaries of the MD simulation cell.

Providing a good description of the heating of ions by hot electrons is also a challenge, since excited electrons cannot spontaneously emit phonons unless the phonons are described quantum mechanically. Both classical MD and Ehrenfest dynamics treat the ionic motion classically, excluding this possibility. A fruitful line of enquiry may be to consider the moment expansion of the ion trajectories around their (Ehrenfest) expectation values [37]. For the present, the state of the art is to invoke the fluctuation-dissipation theorem and convert damped molecular dynamics into Langevin dynamics $[27,9,10]$. This allows the correct average heat flux from electrons to ions to be computed, and thermal energy to 
be returned to the ions as white spectrum noise.

\section{Discussion and Conclusions}

The advance of computing power continually makes possible new types of simulations capable of yielding new insights into old physics. Recently, it has become possible to explore energy exchange processes between metal ions and electrons in unprecedented detail with quantum mechanical simulations: low-energy radiation damage cascades can be directly simulated using time-dependent tightbinding methods, and the heat transfer from fast-moving ions in a system of a few hundred atoms can be computed with TDDFT. These simulations are not bound by simplifying approximations of linearity or electronic homogeneity and so add a new level of realism to models of electronic stopping.

Quantum mechanical simulations have shown that descriptions of electronic stopping based on the idea of electronic friction provide a successful and convenient means of modelling the average power transfer from the ionic subsystem. A simple homogeneous viscous damping coefficient is adequate to model the energy transfer rate, but the accuracy can be improved by making the damping constant dependent on the electron density and by computing the probabilities of specific transitions between bands [28]. The directional nature of the nonadiabatic forces due to electronic heating has also been investigated in detail $[18,34]$, and an approximate form suitable for use in MD simulations proposed. This appears to outperform previous models with little additional computational cost.

As energy is transferred from ions to electrons in countless tiny excitations, the pattern of holes and excited electrons produced by the moving ions appears close to thermal [30]. For this reason, it is meaningful to discuss electronic excitation in terms of a local electronic temperature rise, even where there has been insufficient time to allow true thermalisation to take place. Since the electronic heat capacity is low, it is possible to achieve electronic temperatures of thousands of degrees Kelvin in the vicinity of a keV ion impact. Such high electronic temperatures affect the bonding between atoms [30], implying that it may be necessary to account for temperature dependence in the inter-atomic potentials [38].

Over the next few years we expect to see TDDFT codes confirming the predictions made with simple model Hamiltonians and providing accurate estimates of stopping parameters for use in MD simulations. Existing theories of electronic heat transport and spontaneous phonon emission are more difficult to turn into practical simulation methods, but rapid progress is being made. Fully quantitative simulations of all aspects of the transport and exchange of energy by and between ions and electrons in solids may be only a few years away.

\section{Acknowledgements}

D.R.M. and C.P.R. were supported by EPSRC grant number EP/C524403. 


\section{References}

[1] Gao, F., Bacon, D.J., Flewitt, P.E.J., and Lewis, T.A., The effects of electron-phonon coupling on defect production by displacement cascades in alpha-iron, Modelling and Simulation in Materials Science and Engineering, 6:543-556, (1998).

[2] Kapinos, V.G., and Bacon, D.G., Influence of ion-electron interaction on the formation mechanism of depleted zones in displacement cascades in metals, Phys. Rev. B, 50:13194-13203, (1994).

[3] Nordlund, K., Wei, L., Zhong, Y., and Averback. R.S., Role of electronphonon coupling on collision cascade development in Ni, Pd, and Pt, Phys. Rev. B, 57:R13965-R13968, (1998).

[4] Duvenbeck, A., and Wucher, A., Low-energy electronic excitation in atomic collision cascades: A nonlinear transport model, Phys. Rev. B, 72:165408, (2005).

[5] Duvenbeck, A., Weingart, O., Buss, V., and Wucher, A., Electron promotion and electronic friction in atomic collision cascades, New J. Phys., 9:38, (2007).

[6] Race, C.P., Mason, D.R., Finnis, M.W., Foulkes, W.M.C., Horsfield, A.P. and Sutton, A.P. The treatment of electronic excitations in models of radiation damage in metals, Rep. Prog. Phys., (prov. shed. Oct 2010).

[7] McEniry, E.J., Bowler, D.R., Dundas, D., Horsfield, A.P, Sánchez, C.G. and Todorov, T.N. Dynamical simulation of inelastic quantum transport, J. Phys.: Condens. Matter, 19:196201, (2007).

[8] McEniry, E.J., et al. Modelling non-adiabatic processes using Correlated Electron-Ion Dynamics, Eur. Phys. J. B, (prov. shed. Sept 2010).

[9] Rutherford, A.M. and Duffy, D.M., The effect of electron-ion interactions on radiation damage simulations, Journal of Physics: Condensed Matter, 19:496201, (2007).

[10] Duffy, D.M. and Rutherford, A.M., Including the effects of electronic stopping and electron-ion interactions in radiation damage simulations, Journal of Physics: Condensed Matter, 19:016207, (2007).

[11] Ritchie, R.H., Interaction of charged particles with a degenerate FermiDirac electron gas, Phys. Rev., 114:644-654, (1959).

[12] Lindhard, J., On the properties of a gas of charged particles, Mat. Fys. Medd. K. Dan. Vidensk. Selsk., 28:8, (1954).

[13] le Page, J., Mason, D.R., and Foulkes, W.M.C. The Ehrenfest approximation for electrons coupled to a phonon system, J. Phys.: Condens. Matter, 20:125212, (2008). 
[14] Todorov, T.N. Time-dependent tight binding, J. Phys.: Condens. Matter, 13:10125-10148, (2001).

[15] Car, R. and Parrinello, M., Unified Approach for Molecular Dynamics and Density-Functional Theory, Phys. Rev. Lett., 55:2471-2474, (1985).

[16] Lindenblatt, M., Pehlke, E., Duvenbeck, A., Rethfeld, B., and Wucher, A., Kinetic excitation of solids: The concept of electronic friction, Nucl. Instr. Meth. Phys. Res. B, 246:333-339, (2006).

[17] Pruneda, J.M., Sanchez-Portal, D., Arnau, A., Juaristi,J.I. and Artacho, E., Electronic Stopping Power in LiF from First Principles, Phys. Rev. Lett., 99:235501, (2007).

[18] Mason, D.R., et al. Electronic damping of atomic dynamics in irradiation damage of metals, J. Phys.: Condens. Matter, 19:436209, (2007).

[19] Sutton, A.P., Todorov, T.N., Cawkwell M.J. and Hoekstra, J. A simple model of atomic interactions in noble metals based explicitly on electronic structure, Phil. Mag. A, 81:1833-1848, (2001).

[20] Sutton, A.P., Finnis, M.W., Pettifor,D.G. and Ohta, Y. The Tight Binding bond model, J. Phys. C, 21:35-66, (1988).

[21] Finnis, M.W. and Sinclair, J.E. A simple empirical N-body potential for transition metals, Phil. Mag.A, 50:45-55, (1984).

[22] Firsov, O.B., A quantitative interpretation of the mean electronic excitation energy in atomic collisions, Soviet Physics JETP, 36:1076, (1959).

[23] Lindhard, J. and Scharff, M., Energy dissipation by ions in the keV region, Phys. Rev., 124:128-130, (1961).

[24] Finnis, M.W., Agnew, P., and Foreman, A.J.E., Thermal excitation of electrons in energetic displacement cascades, Phys. Rev. B, 44:567-574, (1991).

[25] Nordlund, K., Ghaly, M., Averback, R.S., Caturla, M., Diaz de la Rubia T. and Tarus, J., Defect production in collision cascades in elemental semiconductors and fcc metals, Phys. Rev. B, 57:7556-7570, (1998).

[26] Ziegler, J.F., Biersack, J.P. and Littmark, U., The stopping and range of ions in solids, Pergamon, (1985).

[27] Caro, A. and Victoria, M., Ion-electron interaction in molecular-dynamics cascades, Phys. Rev. A, 40:2287-2291, (1989).

[28] le Page, J., Mason, D.R., Race,C.P. and Foulkes, W.M.C. How good is damped molecular dynamics as a method to simulate radiation damage in metals?, New J. Phys., 11:013004, (2009). 
[29] Duvenbeck, A., Weingart, O., Buss, V., and Wucher, A., The role of electronic friction of low-energy recoils in atomic collision cascades, $\mathrm{Nucl}$. Instr. Meth. Phys. Res. B, 258:83-86, (2007).

[30] Race, C.P., Mason, D.R., and Sutton, A.P. Electronic excitations and their effect on the inter-ionic forces in simulations of radiation damage in metals, J. Phys.: Condens. Matter, 21:171502, (2009).

[31] Lin, Z.B., and Allen, R.E., Ultrafast equilibration of excited electrons in dynamical simulations, Journal of Physics: Condensed Matter, 21:485503, (2009).

[32] Campillo, I., Pitarke, J.M., and Eguiluz, A.G., Electronic stopping power of aluminum crystal, Phys. Rev. B, 58:10307-10314, (1998).

[33] Duvenbeck, A., Weidtmann, B., Weingart, O., and Wucher, A., Modeling hot-electron generation induced by electron promotion in atomic collision cascades in metals, Phys. Rev. B, 77:245444, (2008).

[34] Race, C.P., Mason, D.R., and Sutton, A.P. An improved model of interatomic forces for large simulations of metals containing excited electrons, New J. Phys., (to appear 2010).

[35] Dubi, Y. and Di Ventra, M., Fourier's law: Insight from a simple derivation, Phys. Rev. E., 79:042101, (2009).

[36] Michel, M., Mahler, G., and Gemmer, J., Fourier's Law from Schrödinger Dynamics, Phys. Rev. Lett., 95:180602, (2005).

[37] Horsfield, A.P., Bowler, D.R., Fisher, J., Todorov, T.N. and Sánchez, C.G., Correlated electron-ion dynamics: the excitation of atomic motion by energetic electrons, Journal of Physics: Condensed Matter, 17:4793-4812, (2005).

[38] Khakshouri, S., Alfè, D., and Duffy, D.M., Development of an electrontemperature-dependent interatomic potential for molecular dynamics simulation of tungsten under electronic excitation, Phys. Rev. B, 78:224304, (2008). 